\title{
The effect of 1-year mean step count on the change in the atherosclerotic cardiovascular disease risk calculation in patients with high cardiovascular risk: a sub-study of the LIGHT randomized clinical trial
}

\author{
Mert Illker Hayıroğlu', Tufan Çınar², Göksel Çinier', Arif Karakaya², Muhammet Yıldırım², Başak Çakır Güney²,

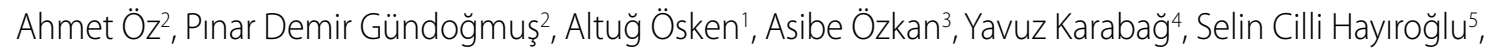 \\ Mustafa Kaplan², Can Altundaş', Ahmet Illker Tekkeşin'
}

\begin{abstract}
'Department of Cardiology, Dr. Siyami Ersek Thoracic and Cardiovascular Surgery Training and Research Hospital, Istanbul, Turkey 2Department of Cardiology, Haydarpasa Sultan II. Abdulhamid Han Training and Research Hospital, Istanbul, Turkey

${ }^{3}$ Department of Medical Nursing, Dr. Siyami Ersek Thoracic and Cardiovascular Surgery Training and Research Hospital, Istanbul, Turkey

${ }^{4}$ Department of Cardiology, Kafkas University Faculty of Medicine, Istanbul, Turkey

${ }^{5}$ Department of Rheumatology, Marmara University Faculty of Medicine, Istanbul, Turkey
\end{abstract}

Correspondence to:

Mert Illker Hayıroğlu, MD, PhD, Assoc. Prof Department of Cardiology, Dr. Siyami Ersek Thoracic and Cardiovascular Surgery Training and Research Hospital,

Evsen Street 11/10 Goztepe Kadıkoy, Istanbul, Turkey, phone: +90 53571548 08, e-mail:

mertilkerh@yahoo.com

Copyright by the Author(s), 2021

Kardiol Pol. 2021;

79 (10): 1140-1142;

DOI: 10.33963/KP.a2021.0108

Received:

August 5, 2021

Revision accepted:

September 5, 2021

Published online:

September 6, 2021

\section{INTRODUCTION}

Increased physical activity has been one of the main recommendations for both the primary and secondary prevention of cardiovascular diseases [1]. Consistent physical activity has already proven its protective and therapeutic value in numerous conditions, such as metabolic syndrome and cardiovascular diseases $[2,3]$. Despite the well-defined beneficial effects, physical activity is still not performed at the desired level, especially for the primary prevention of the disease in patients with multiple cardiovascular risk factors. Smartphone applications offer an up-and-coming infrastructure to potentiate suggestions regarding physical activity, especially about a daily step count [4].

The atherosclerotic cardiovascular disease (ASCVD) risk score has been widely approved for patient follow-up and to guide primary prevention strategies [1]. It is invaluable in decreasing or maintaining a steady state in these risk estimator scores. However, there are no daily-step-count recommendations particularly in patients at high cardiovascular risk. Thus, we aim to assess the effect of the 1-year mean step count (MSC) on the 1-year change in ASCVD risk scores in patients at high risk of cardiovascular disease.

\section{METHODS}

Our study was a sub-study of the lifestyle intervention using mobile technology in patients with high cardiovascular risk: a pragmatic randomized clinical (LIGHT) trial conducted in a tertiary hospital between February 2018 and March 2020 [5] (NCT03397849). All patients were randomized into usual care or usual care plus the lifestyle intervention using mobile technology. The randomization and patient enrolment were described previously [6]. The study population comprised 242 patients allocated into usual care plus intervention in the LIGHT trial. All patients were given information about a mobile application specially designed for that study, which included sections for step counts, weight, blood pressure, and diet. Wristbands (Xiaomi Mi Band 2; Beijing Xiaomi Technology Co., Beijing, China) were used to record the patients' daily step counts. The daily data were monitored from the main study server and defined automatic messages were delivered according to the study design [6]. Patients were followed up for 12 months after randomization. Demographic characteristics associated with clinical data and ASCVD risk scores were recorded prospectively at baseline and the 1-year follow-up. A change in ASCVD risk score $(\triangle \mathrm{ASCVD})$ between randomization 
and the final stage was also determined. The study population was divided into two groups according to their $\triangle$ ASCVD risk scores as follows: Group 1, the patients with a decrease in the ASCVD risk score at 1-year; Group 2, the patients with an increase in the ASCVD risk score at 1-year (Supplementary material, Table S1). The relationship between the mean daily step count at 1-year and the ASCVD risk score was comprehensively analyzed. The 1-year mean daily step count was calculated by dividing the total number of steps recorded by the number of data entry days. The investigation was approved by the local ethics committee.

\section{Statistical analysis}

Data were presented as mean (standard deviation [SD]) for normally distributed data and as median (interquartile range [IQR]) for continuous variables that were not normally distributed. Categorical data were presented as number (\%). The Mann-Whitney U test was applied for comparisons of the data that were not normally distributed. Categorical data were analyzed with Pearson's chi-square test. Univariable and multivariable logistic regression analyses were performed to determine independent predictors of a decrease in the ASCVD risk score. Variables that could be predictors of a decrease in the ASCVD risk score were entered into the univariable analysis. Variables with a $P$-value $<0.1$ in univariable regression were incorporated into the multiple logistic regression analysis. The results of the regression analysis were shown as odds ratios with $95 \%$ confidence intervals. Cut-off values of the MSC at 1 year to predict a decrease in ASCVD risk scores with the highest sensitivity and specificity were calculated by nonparametric receiver operating characteristics (ROC) curve analysis. To introduce the association between MSC at 1-year and $\triangle A S C V D$, a correlation analysis was performed using the Spearman test. $P$ values of $<0.05$ were considered significant. Data were analyzed with the Statistical Package for Social Sciences software, version 22.0 (SPSS; IBM, Armonk, NY, USA).

\section{RESULTS AND DISCUSSION}

A total of 242 patients were enrolled (mean age 58.3 [7.1] years; $51.2 \%$ male) in the study population and evaluated according to the changes in their ASCVD risk scores for 1 year after enrolment. Data input frequency was $75 \%$ for the least compliant patient, and data entry compliance was similar between the patients with and without a decrease in ASCVD risk scores at 1 year $(P=0.595)$.

The univariable analysis revealed age, systolic and diastolic blood pressure, hypertension, diabetes mellitus, left atrial volume index, HDL-cholesterol, $\mathrm{HbA1c}$, angiotensin-converting enzyme (ACE) inhibitors or angiotensin receptor blockers (ARBs), and MSC over 1 year as predictors of a decrease in the ASCVD risk score at 1 year. In the multivariable analysis, age (odds ratio [OR], 0.908; $\mathrm{Cl}$, 0.849-0.971; $P=0.005)$, diabetes mellitus (OR, 0.200; $\mathrm{Cl}$, 0.076-0.527; $P=0.001$ ), use of ACE inhibitors or ARBs (OR, $3.422 ; \mathrm{Cl}, 1.320-8.870 ; P=0.011)$, and MSC over 1 year $(\mathrm{OR}$, 1.103; $\mathrm{Cl}, 1.071-1.136 ; P<0.001)$ were determined to be independent factors that predict a decrease in the ASCVD risk score.

The Spearman rank correlation proved that 1-year MSC is correlated with the $\triangle A S C V D$ risk score. (Rho: -0.523 ; $P<0.001$ ) (Figure 1A). ROC analysis showed that the best cut-off value of the 1-year MSC to predict a decrease in $\triangle A S C V D$ was 7250 with $81 \%$ sensitivity and $82 \%$ specificity (area under the curve: $0.88 ; 95 \% \mathrm{Cl}, 0.84-0.93 ; P<0.001$ ) (Figure 1B).

Our investigation demonstrated that daily step count may play an important role in the management of a cardiovascular disease. In addition, age, diabetes mellitus, and the use of ACE inhibitors or ARBs as baseline medications were determined to be independent predictors of a decrease in the ASCVD risk score at the 1-year follow-up. The correlation between 1-year MSC and the $\triangle$ ASCVD risk score also emphasized the significance of daily step count in the primary prevention strategies for patients at high cardiovascular risk.
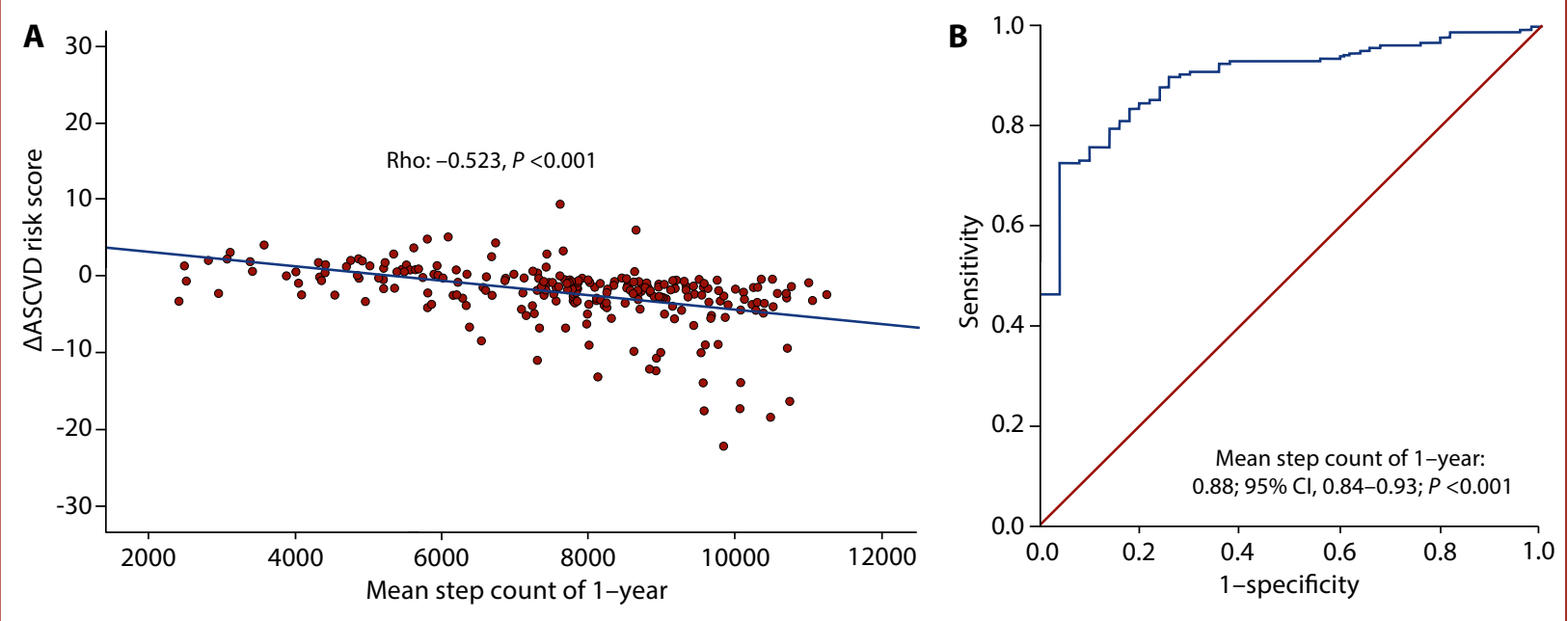

Figure 1. A. The correlation between 1-year mean daily step count and $\triangle A S C V D$ and $\triangle S C O R E$ risk calculations is presented with a scatter-dot analyses. B. ROC analysis showing the best cut-off value of the 1-year mean step count to predict a decrease in $\triangle \mathrm{ASCVD}$ and $\triangle \mathrm{SCORE}$ 
Mobile health applications have already demonstrated their instrumentality and practicality in the screening of atrial fibrillation and hypertension $[7,8]$. Our results may have an indispensable value for setting targets for patients considering daily step counts to decrease their long-term cardiovascular risk. Several daily step count targets have been recommended for promoting healthy lifestyles in patients without cardiovascular diseases [9], and completing 10000 steps per day has been shown to be a rational objective for healthy people [10]. However, there is a lack of evidence for determining a cut-off value for decreasing well-accepted risk scores such as ASCVD. This should be emphasized as one of the most important strengths of our investigation. However, the quality and target of the therapy in patients with hypertension and diabetes may have an important role in risk reduction for patients with high cardiovascular risk similar to our study population [11]. Thus, a novel strategy based on the COMPASS trial could be used to properly evaluate both the effect of the medications and MSC on the change in the cardiovascular risk [12].

Our investigation has several limitations secondary to the study design and implementation OF WHAT AUTHORS UNCLEAR. First, the patients were recruited and followed up in a single center. Second, the data entry was not 100\%, but the compliance was provided with the aid of automatic messages and individualized phone calls inviting the patients for an outpatient visit. $83 \%$ data input frequency was accomplished using the methods in the study design; however, it may not represent correctly the 1-year follow-up due to missing data. Third, the study was implemented with individually provided data; however, if the data had not been compatible, they were verified through invitations to outpatient visits. Fourth, the association between MSC and the change in the 1-year ASCVD risk score does not prove causality.

\section{Supplementary material}

Supplementary material is available at https://journals. viamedica.pl/kardiologia_polska.

\section{Article information}

Conflict of interest: None declared.

Open access: This article is available in open access under Creative Common Attribution-Non-Commercial-No Derivatives 4.0 International (CC BY-NC-ND 4.0) license, allowing to download articles and share them with others as long as they credit the authors and the publisher, but without permission to change them in any way or use them commercially. For commercial use, please contact the journal office at kardiologiapolska@ptkardio.pl.

How to cite: Hayıroğlu MI, ÇınarT, Çinier G, et al. The effect of 1-year mean step count on the change in the ASCVD risk calculation in patients with high cardiovascular risk: a sub-study of the LIGHT randomized clinical trial. Kardiol Pol. 2021; 79(10): 1140-1142, doi: 10.33963/KP.a2021.0108.

\section{REFERENCES}

1. Piepoli MF, Hoes AW, Agewall S, et al. ESC Scientific Document Group. 2016 European Guidelines on cardiovascular disease prevention in clinical practice: The Sixth Joint Task Force of the European Society of Cardiology and Other Societies on Cardiovascular Disease Prevention in Clinical Practice (constituted by representatives of 10 societies and by invited experts)Developed with the special contribution of the European Association for Cardiovascular Prevention \& Rehabilitation (EACPR). Eur Heart J. 2016; 37(29):2315-2381, doi: 10.1093/eurheartj/ehw106, indexed in Pubmed: 27222591.

2. Schmidt MD, Cleland VJ, Shaw K, et al. Cardiometabolic risk in younger and older adults across an index of ambulatory activity. Am J Prev Med. 2009; 37(4): 278-284, doi: 10.1016/j.amepre.2009.05.020, indexed in Pubmed: 19765498.

3. Vasankari V, Husu P, Vähä-Ypyä H, et al. Association of objectively measured sedentary behaviour and physical activity with cardiovascular disease risk. Eur J Prev Cardiol. 2017; 24(12): 1311-1318, doi: 10.1177/2047487317711048, indexed in Pubmed: 28530126.

4. Romeo A, Edney S, PlotnikoffR, et al. Can smartphone apps increase physical activity? Systematic review and meta-analysis. J Med Internet Res. 2019; 21(3): e12053, doi: 10.2196/12053, indexed in Pubmed: 30888321.

5. Tekkeşin Al, Hayıroğlu Mi, Çinier G, et al. Lifestyle intervention using mobile technology and smart devices in patients with high cardiovascular risk: A pragmatic randomised clinical trial. Atherosclerosis. 2021; 319: 21-27, doi: 10.1016/j.atherosclerosis.2020.12.020, indexed in Pubmed: 33465658.

6. Tekkesin I, Çinier G, Hayıroğlu M, et al. Rationale and design of lifestyle intervention using mobile technology in patients with high cardiovascular risk: A pragmatic randomized clinical trial. Turk Kardiyol Dern Ars. 2020; 48(2): 149-157, doi: 10.5543/tkda.2019.69494, indexed in Pubmed: 32147654.

7. Chan PH, Wong CK, Poh YC, et al. Diagnostic performance of a smartphone-based photoplethysmographic application for atrial fibrillation screening in a primary care setting. J Am Heart Assoc. 2016; 5(7): e003428, doi: 10.1161/JAHA.116.003428, indexed in Pubmed: 27444506.

8. Parati G, Pellegrini D, Torlasco C. How digital health can be applied for preventing and managing hypertension. Curr Hypertens Rep. 2019; 21(5): 40, doi: 10.1007/s11906-019-0940-0, indexed in Pubmed: 31011866.

9. Ishikawa-Takata K, Tabata I. Exercise and physical activity reference for health promotion 2006 (EPAR2006). J Epidemiol. 2007; 17(5): 177, doi: 10.2188/jea.17.177, indexed in Pubmed: 17827865.

10. Tudor-Locke C, Craig CL, Brown WJ, et al. How many steps/day are enough? For adults. Int J Behav Nutr Phys Act. 2011; 8: 79, doi: 10.1186/1479-5868-8-79, indexed in Pubmed: 21798015.

11. Burnier M. Controversies in the management of patients with arterial hypertension. Kardiol Pol. 2019; 77(10): 902-907, doi: 10.33963/KP.15002, indexed in Pubmed: 31571674.

12. Kruger PC, Guzik TJ, Eikelboom JW. How can the results of the COMPASS trial benefit patients with coronary or peripheral artery disease in Poland? Kardiol Pol. 2019; 77(7-8): 661-669, doi: 10.33963/KP.14855, indexed in Pubmed: 31144674. 